\title{
Single parenchymal brain cysticercus in the acute encephalitic phase: definition of a distinct form of neurocysticercosis with a benign prognosis
}

\author{
Oscar H Del Brutto
}

\begin{abstract}
Fifty four patients with a single parenchymal brain cysticercus in the acute encephalitic phase were studied to outline the features of this form of the disease. Seizures were the presenting symptom in all cases. Twenty six patients had a single seizure and 28 had several seizures before admission. Neurological examination was normal in 45 patients and showed focal signs in nine. All patients had a single enhancing CT lesion; all but three lesions were $<20 \mathrm{~mm}$. Anticonvulsants were started in every patient. Forty five patients were followed up for 18 (SD 6) months. Thirty seven of these 45 patients received albendazole. Four weeks after the trial, CT showed resolution of lesions in all cases. The remaining eight patients refused albendazole, and CT showed persistence of lesions by 16 weeks in six cases. At the end of the follow up, all patients who received albendazole were free of seizures as opposed to three of eight patients who did not receive the drug. Focal signs improved in the nine patients with these signs (all received albendazole). Recognition of this form of neurocysticercosis permits early treatment with albendazole that greatly improves the prognosis.
\end{abstract}

(F Neurol Neurosurg Psychiatry 1995;58:247-249)

Keywords: cysticercosis; Taenia solium; neurocysticercosis; computed tomography

Neurocysticercosis (NCC) is a pleomorphic parasitic disease that shows different patterns of clinical manifestations in almost every patient. In some cases the disease runs an indolent course characterised by sporadic seizures or mild headache, but in others it may progress with intractable epilepsy, intracranial hypertension, coma, and death. Individual differences in the number and location of lesions within the CNS as well as differences in the degree of the host's immune reaction against cysticerci usually account for this great variability in the severity of disease expression. ${ }^{1}$
Several studies have focused on severe forms of neurocysticercosis. Nevertheless, more benign forms of the disease have not been properly individualised. Because this knowledge is important for therapeutic and prognostic purposes, I conducted this study to outline the features of a relatively benign form of neurocysticercosis characterised by a single parenchymal brain cysticercus in the acute encephalitic phase.

\section{Methods}

Fifty four consecutive patients with a single parenchymal brain cysticercus in the acute encephalitic phase were studied. They represented $23 \%$ of the 236 patients with neurocysticercosis who were evaluated at the Department of Neurology, Luis Vernaza Hospital, Guayaquil, from June 1987 to May 1993. The diagnosis was based on CT findings of a single hypodense parenchymal brain lesion surrounded by oedema and ring-like or nodular enhancement after contrast medium was given. ${ }^{1}$ No patient had evidence of a systemic illness, including tuberculosis, neoplasia, or acquired immunosuppression. These conditions as well as other entities that may also progress with a single enhancing CT lesion, were ruled out by appropriate diagnostic tests including radiographs of the chest, routine metabolic tests, and cerebral angiography or CSF examination in selected cases. The diagnosis of neurocysticercosis was further confirmed in most patients by the disappearance of the brain lesion as the result of a therapeutic course of albendazole. ${ }^{2}$

Medical records were reviewed with special emphasis on clinical manifestations, results of neurological examination, EEG and CT findings, therapeutic approach, and outcome.

\section{Results}

The patients were 34 men and 20 women with a mean age of 35 (range 14 to 65 ) years. Seizures were the presenting symptom in all patients. Twenty six patients had a single seizure and 28 had several seizures from two to 12 weeks before admission. Seizures were simple partial in 37 patients and tonic-clonic generalised in 17 . Twenty nine of the 37 patients with simple partial seizures had 
Contrast enhanced CT of a patient with a single parenchymal brain cysticercus in the acute encephalitic phase located in the frontal lobe, before (left) and four weeks after (right) treatment with albendazole. Note the complete resolution of the brain lesion after treatment. motor symptoms with or without secondary generalisation, five patients had somatosensory symptoms, and three patients had elementary visual manifestations. The neurological examination was normal in 45 patients and showed focal signs in nine. Of these, seven patients had hemiparesis or monoparesis, one patient had hemisensory loss, and the other had a visual field defect. Electroencephalography was performed in 37 patients and showed abnormal findings in nine $(24 \%)$. Among these patients, six had diffuse slowing and three had focal paroxysmal activity.

Computed tomography showed a single parenchymal brain lesion in every case. On plain CT, lesions were hypodense, ill defined, and surrounded by oedema. After contrast medium lesions showed two major patterns of abnormal enhancement, the "ring" pattern in which there was peripheral enhancement of the lesion, and the "nodular" pattern in which there was uniform enhancement of the whole lesion. Forty lesions had the ring pattern, and the remaining 14 had the nodular pattern of abnormal enhancement. Fifty one lesions were $<20 \mathrm{~mm}$ in size, and the other three measured from 20 to $30 \mathrm{~mm}$. All lesions were located within the cerebral hemispheres, and most of them were near to the cortical surface. There were 30 right sided and 24 left sided lesions. Lesions were located in the parietal lobe in 28 patients, the frontal lobe in 17, the occipital lobe in six, and the temporal lobe in two. The remaining lesion was located in the basal ganglia.

Anticonvulsant treatment with either phenytoin (300 to $400 \mathrm{mg} /$ day) or carbamazepine ( 600 to $800 \mathrm{mg} /$ day) was started in every patient. Nine patients were lost to follow up. The remaining 45 patients were followed up for three months to four years (mean follow up 18 (SD 6) months). Thirty seven of these 45 patients were treated with albendazole at daily doses of $15 \mathrm{mg} / \mathrm{kg}$. The first 12 patients received albendazole for 30 days, and the last 25 patients received the drug for eight days. Computed tomography performed four weeks after the trial showed resolution of lesions in all cases, including complete disappearance of the brain lesion in 31 patients (figure) and a residual calcification in six. Eight patients refused albendazole treatment, and CT showed persistence of the brain lesion by 16 weeks in six cases. At the end of the follow up, all patients who received albendazole were free of seizures as opposed to three of eight patients who did not receive the drug $(100 \% v 37.5 \%, \mathrm{p}<0.0005)$. Focal signs improved in the nine patients (all received albendazole).

\section{Discussion}

Parenchymal brain cysticerci in the acute encephalitic phase have been recognised since the first reports of CT in patients with neurocysticercosis. ${ }^{3}$ These lesions were described as focal low densities surrounded by oedema and ring-like enhancement after giving contrast medium. Thereafter, RodriguezCarbajal et $a l^{4}$ correlated this CT finding with a particular stage in the natural evolution of cysticerci called "colloidal stage", in which the parasites begin to degenerate as the result of an intense immune reaction from the host. According to the authors, the abnormal enhancement of these lesions was related to a breakdown in the blood-brain barrier caused by the inflammatory reaction around dying cysticerci.

The clinical manifestations and the prognosis of patients with parenchymal brain cys-
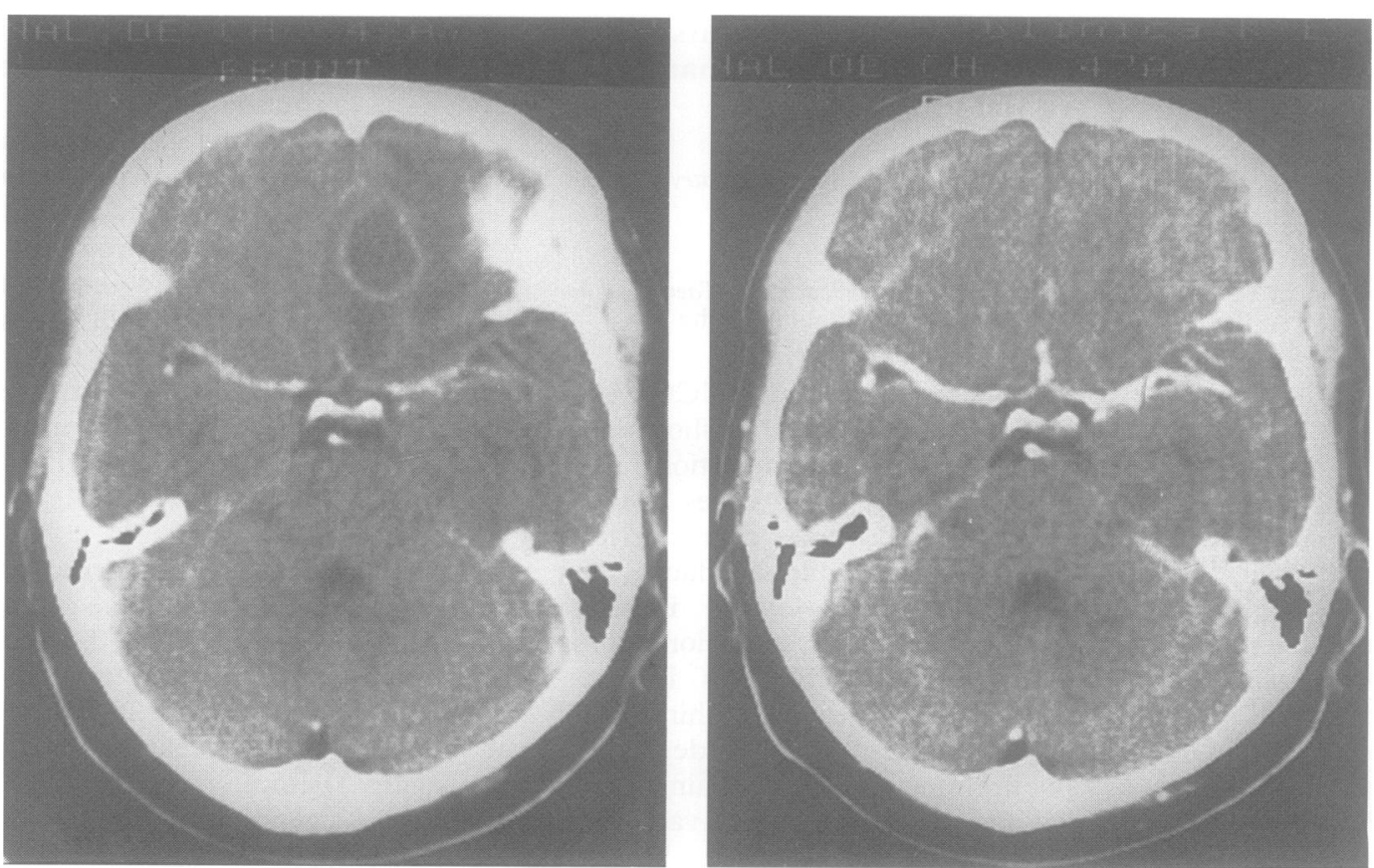
ticerci in the acute encephalitic phase have not been properly characterised. Whereas some authors have considered that this is a benign form of neurocysticercosis, others believe that such patients may be severely ill or may even die as the result of the inflammatory reaction around parasites. The main factor accounting for the differences in the prognosis of this form of the disease has been the lack of uniformity among the patients included in each series. In recent years it became apparent that the prognosis of patients with multiple parenchymal brain cysticerci in the acute encephalitic phase may be ominous. This form of the disease, called cysticercotic encephalitis, represents a severe form of neurocysticercosis in which the nervous system is harmed by the intense immune reaction mounted by the host against a massive cysticerci infestation of the brain parenchyma. ${ }^{1}$ Anticysticercal drugs are contraindicated in patients with cysticercotic encephalitis because they may exacerbate the inflammatory response within the brain parenchyma.

On the other end of the spectrum of disease severity are the patients with a single parenchymal brain cysticercus in the acute encephalitic phase described in this series. Seizures were the main complaint in all cases and most patients had a normal neurological examination. Anticysticercal drugs were actually indicated in such patients as their use favoured the control of seizures with antiepileptic drugs. This study and a recent paper by Vazquez and Sotelo ${ }^{5}$ show that the prognosis of patients with single parenchymal brain cysts in the acute encephalitic phase is significantly better after treatment with albendazole than when the disease is left untreated. Another indication for the routine use of anticysticercal drugs in these patients is the hastening in the disappearance of lesions that such treatment produces (figure). The differential diagnosis of single enhancing CT lesions is difficult as parasitic cysts, tubercu- lomas, mycotic granulomas, brain tumours, and vascular malformations may present similar findings. Unfortunately, neither a complete integration of data from clinical manifestations, CSF analysis, serological tests, and neuroimaging studies provide clues to the correct diagnosis in some cases. Two recent preliminary reports ${ }^{26}$ suggested that albendazole given to every patient with a single enhancing CT lesion is a useful tool for the diagnosis of cysticercosis which, in many areas of the world, is the leading cause of this CT finding. This report further confirmed these findings, providing a rational argument for the use of albendazole in patients with colloidal cysticerci.

From this series it is clear that single parenchymal brain cysticerci in the acute encephalitic phase represent a distinct form of neurocysticercosis with benign prognosis. These patients mostly have seizures as the leading or sole manifestation of the disease and the neurological examination is usually normal. Electroencephalograms are usually normal or may show non-specific abnormalities. Early treatment with albendazole facilitates the diagnosis and improves the prognosis by allowing a better control of seizures.

This work was presented in abstract form at the 46th Annual Meeting of the American Academy of Neurology, Washington, DC May 1994

1 Del Brutto OH, Sotelo J. Neurocysticercosis: an update. Rev Infect Dis 1988;10:1075-87.

2 Del Brutto $\mathrm{OH}$. The use of albendazole in patients with single lesions enhanced on contrast CT. N Engl ₹ Med 1993;328:356-7.

3 Mervis B, Lotz JW. Computed tomography (CT) in parenchymatous cerebral cysticercosis. Clin Radiol 1980; 31:521-8.

4 Rodriguez-Carbajal J, Salgado P, Gutierrez-Alvarado R, Escobar-Izquierdo A, Aruffo C, Palacios E. The acute encephalitic phase of neurocysticercosis: computed tomographic manifestations. AfNR Am $\mathfrak{7}$ Neuroradiol tomographic

5 Vazquez V, Sotelo J. The course of seizures after treatment for cerebral cysticercosis. N Engl f Med 1992;327: 696-701.

6 Rajshekhar V. Albendazole therapy for persistent, solitary cysticercus granulomas in patients with seizures. Neurology 1993;43:1238-40. 